\title{
Obstetric Vesicouterine Fistulas: About 26 Cases Managed at Cocody's University Hospital (Abidjan-Cote d'Ivoire)
}

\author{
Vedi Andre Loue ${ }^{1,2, *}$, Mamadou Salia Traore ${ }^{1}$, Kouadio Achille Koffi ${ }^{2}$, Cassou Roland Adjoby ${ }^{2}$, \\ Arthur Didier Kouame ${ }^{2}$, Akpa Yehi Gbary ${ }^{2}$, Ehouman Serge Boni ${ }^{2}$ \\ ${ }^{1}$ Paris Descartes University, Sud Francilien Hospital Center (Woman-Mother-Child Pole), Paris, France \\ ${ }^{2}$ Felix Houphouet-Boigny' University, Faculty of Medical Sciences (Mother and Child Department, University Hospital of Cocody), Abidjan, \\ Cote d'Ivoire
}

Email address:

drlouevedi@yahoo.fr (V. A. Loue)

${ }^{*}$ Corresponding author

\section{To cite this article:}

Vedi Andre Loue, Mamadou Salia Traore, Kouadio Achille Koffi, Cassou Roland Adjoby, Arthur Didier Kouame, Akpa Yehi Gbary, Ehouman Serge Boni. Obstetric Vesicouterine Fistulas: About 26 Cases Managed at Cocody's University Hospital (Abidjan-Cote d'Ivoire). Journal of Gynecology and Obstetrics. Vol. 5, No. 1, 2017, pp. 20-24. doi: 10.11648/j.jgo.20170501.13

Received: January 26, 2017; Accepted: February 24, 2017; Published: March 2, 2017

\begin{abstract}
The aim of this work is to recall the etiopathogenic, diagnostic and therapeutic aspects of vesicouterine fistulas. This is a prospective study of 26 cases of obstetric vesicouterine fistula cared for in Cocody University Hospital Center (Abidjan, Cote d'Ivoire) between 07/09/11 and 24/04/2015. Early in the disease, patients were all in reproductive age with a different obstetrical history. The mean duration of the signs before diagnosis was 6 years. Etiologies are largely dominated by caesarean sections (69\%) which four iteratives. The classic Youssef's triad syndrome had represented 42.3\%. Both hysterography and cystography were sufficient to establish the diagnosis of vesicouterine fistula in all cases. All patient underwent transperitoneal laparotomy repair. Peritoneal patch had been necessary in two cases and in four cases a hysterectomy was performed. Functional outcome was satisfactory. Vesicouterine fistula is a rare obstetric complication and very often iatrogenic. The good prognosis after repairing should not make us forget that this pathology seriously affects the quality of life of the patient. There must be a permanent and quality prevention.
\end{abstract}

Keywords: Obstetric Fistulas, Vesicouterine Fistulas, Youssef's Syndrome

\section{Introduction}

Urogenital fistula is an abnormal communication between the urinary tract and the gynecological apparatus. As a consequence of poverty, lack or inadequacy of emergency obstetric care, their actual number is difficult to know because it is a hidden condition. It is estimated that it now affects about 2 million women worldwide with an incidence of 50,000 to 100,000 new cases per year [1]. It is accompanied by a recto-vaginal fistula in $6-24 \%$ according to different studies. Sub-Saharan Africa is paying the heavy toll, thus constituting a major public health problem in our countries [1-3]. The vesicouterine fistulas that communicate bladder and uterus are the rarest $(1-5 \%)$ of urogenital fistulas but appear to be increasing linked to the persistence and / or to the increase of risk factors. The medical, physical and social consequences of fistula prove the particular gravity of this obstetric complication and deserve vigorous action to eradicate it.

The aim of this work is to recall the etiopathogenic, diagnostic and therapeutic aspects of this pathology in order to continue stimulating a real preventive action.

\section{Methods}

This is a prospective study of 26 cases of obstetric vesicouterine fistula. Patients were cared for in the gynecology department of the Cocody University Hospital Center (Abidjan, Cote d'Ivoire) between 07/09/11 and 24/04/2015 in the context of the free management of obstetric complications initiated by our country. A prospective data collection sheet had been prepared for this purpose and data 
started to be collected prospectively in the period of study. In this study, several parameters were taken into account, including the age of the patients at the onset of symptoms, the age of the patients at the time of diagnosis and management, the obstetric history of each patient, the distance between the place of residence and the place of delivery, the duration of labor, the duration of the fistula before diagnosis, the clinical and therapeutic aspects of the affection. Patients' management was done by a multidisciplinary team. Immediate postoperative monitoring concerned the detection of infectious signs, drainage of urine, the quality of micturition, recurrent urinary incontinence and the obstetric future of some patients.

\section{Results}

\section{(a)-Patients general characteristics}

At the onset of symptoms, the patients were all young in the reproductive age (mean age 32 years, extremes of 21 and 42 years). Twelve patients lived as a couple and fourteen were single. At the time of repair, the average age was 37 years (extremes of 26 and 64 years) and no patients lived as a couple. Six patients were menopausal or near menopausal. In the majority of cases, they had an agricultural profession and were illiterate, living in rural areas.

\section{(b)-Obstetrical history}

-Concerning the antecedents: the mean parity was 6.2 (extremes of 1 and 12) with an average number of vaginal births of 5.1 (extremes of 1 and 10). Two patients had a scarred uterus; one patient had a history of 2 cesareans and another patient a history of three cesareans.

-For the delivery giving rise to fistula: the patients gave birth in a university hospital center $(n=4)$, in a regional hospital center $(n=12)$, in a general hospital $(n=6)$ and at home $(n=4)$. The deliveries' modes are summarized in Table 1. Delivery was performed by an obstetrician $(n=16)$, by a midwife $(n=6)$, and by a traditional birth attendant $(n=4)$. Patients who gave birth in a medical center had traveled more than $15 \mathrm{~km}(\mathrm{n}=16)$ and less than $5 \mathrm{~km}(\mathrm{n}=6)$. The duration of labor was less than 24 hours $(\mathrm{n}=8)$, between 1 and 3 days $(\mathrm{n}=16)$ and more than 3 days $(\mathrm{n}=2)$. At birth, newborns were alive in 6 cases and deaths in 20 cases.

Table 1. Birth modes giving rise to the fistula.

\begin{tabular}{lll}
\hline Birth modes & Number & $\mathbf{\%}$ \\
\hline Vaginal birth & 5 & 19.2 \\
Vaginal birth with forceps & 2 & 7.7 \\
Vaginal birth after Caesarean section & 1 & 3.9 \\
Caesarean section & 14 & 53.8 \\
Iterative Caesarean section & 4 & 15.4 \\
Total & 26 & 100 \\
\hline
\end{tabular}

\section{(c)-Diagnostic aspects}

The mean duration of the signs before diagnosis was 6 years (extremes of 2 months and 22 years). Clinical signs or reasons for consultation have been summarized in Table 2 . Isolated urinary leaks or associated with another signs dominate the panel, followed by the classic Youssef's triad.
During the speculum examination, bladder filling with physiological saline supplemented with blue methylene revealed blue in the cervix in 21 cases. Radiographic exploration has been carried out in all cases given the context of free admission according to the possibilities of our university hospitals. Hysterosalpingography and retrograd urethocystography disclosed the communication between bladder and uterine cavity, confirming the vesicouterine fistula in all cases. The hysterosalpingography also revealed bilateral tubal obstruction in one case and unilateral tubal obstruction in another case. In intravenous urography, the upper urinary tract was normal in all cases. Cystoscopy was performed with a didactic aim in 14 patients and revealed the fistula in 5 cases.

Table 2. Observed symptoms or reasons for consultation.

\begin{tabular}{lll}
\hline Reasons for consultation & Number & $\mathbf{\%}$ \\
\hline Isolated and permanent urinary leak & 10 & 38.5 \\
Urinary leak and cyclical hematuria & 4 & 15.4 \\
Menouria, amenorrhea and urinary continence & 11 & 42.3 \\
Urinary incontinence and faecal leak* & 1 & 3.8 \\
Total & 26 & 100 \\
\hline
\end{tabular}

* This patient presented with both vesicouterine and rectovaginal fistula.

\section{(d)-Treatment and prognosis}

The waiting period for each patient before treatment consisted essentialy of the preoperative preparation, the treatment of indirectly associated pathologies (anemia, malnutrition, infection) and the treatment of other general diseases. All patient underwent transperitoneal laparotomy repair as a first attempt surgical treatment. Bladder and uterus were dissected and separated. The lesion was detected and excised following the opening of the pathological pathway. Uterine and bladder sutures were made using resorbable thread. Peritoneal patch was given between uterus and bladder as necessary in two cases. In four cases a hysterectomy with bladder suture was performed. The rectovaginal fistula was treated by transvaginal approach. The control test with methylene blue to check the vesical seal (continence) was normal for all patients. Urine drainage by bladder catheterization was done for at least 21-28 days. The closure of the fistula was obtained in all cases, of which 3 after a second operation. Counseling prior to discharge included time to resume sexual intercourse, family planning counseling, the importance of future deliveries in hospitals, and encouragement of social reintegration. To date, among the patients operated, eight had been able to lead a pregnancy.

\section{Discussion}

Among the urogenital fistulas, vesicovaginal fistulas are the most frequent $[1,2]$, followed by uretero-vaginal fistulas $(5-8 \%)$. Vesico-uterine fistulas represent a rare variety of urogenital fistulas (1-5\%). The actual incidence in subSaharan Africa of urogenital fistulas in general and vesicouterine fistulas in particular is not well known. 
However with the evolution of caesarean rates which tend to know a real inflation today, it is possible that their incidence will increase significantly. This is a common condition in Cote d'Ivoire [4]. Given this observation, it seems important, even imperative, to recall the etiopathogenesis of this shameful obstetric complication in order to require real awareness.

The intimate proximity in the pelvis of the genital and urinary apparatus exposes them in common to obstetrical trauma and errors of surgical techniques. In the past, obstetric complications like prolonged obstructed labor, coupled with a lack of medical attention were more common causes of vesicouterine fistulas $[5,6]$. In developped countries, with much better obstetrics facilities the causative factors are mostly associated with gynecologic and pelvic surgery [7]. Rare causes have been reported in the literature: the fistula may be secondary to vaginal birth on scarred uterus, it can also occur following high vaginal forceps-aided delivery, external cephalic version, curettage or manual removal of the placenta, placenta percreta [8], uterine rupture due to obstructed labor, uterine artery embolization, migration of an intrauterine device secondary to an actinomycotic infection [9], vesical tuberculosis, cervical circling [10], and brachytherapy for cervix carcinoma [11]. But currently, however, there has been an increase in fistulas cases resulting from caesarean section [12]. While most obstetric fistula are vesicovaginal, the vesicouterine type is recognized to occur most often secondary to caesarean delivery $[12,13]$. In the present study, etiologies of fistulas are obstetric, largely dominated by caesarean sections $(69 \%)$ confirming the data of the literature, then come the consequences of the obstructive labor (Table 1).

More than half of the vesicouterine fistulas in this study are secondary to a caesarean section, which is certainly complicated by an unknown bladder wound; especially since it is an iterative caesarean section $(15.4 \%)$ where the uterine detachment is often difficult or an emergency caesarean section (53.8\%) after a long obstructed labor ( 18 out of 26 patients had more than one day of labor) with oedematized tissues and where the eagerness of the surgical procedure exposes to many errors, especially since it is in our context for the most part young surgeons. Three mechanisms can be responsible for the creation of a vesico-uterine fistula [14, 15]: the first mechanism is represented by the operative trauma of the bladder. This trauma is the result of a laborious vesicouterine detachment such as is observed in the iterative caesarean sections or skewering the bladder during uterine suture. The second mechanism is represented by the prolonged compression by the fetal mobile on the bladder and uterine wall against the pubic symphysis generating ischemia, the fistula resulting from the fall of bedsores and the inter-vesicouterine abscess after caesarean section is the third mechanism retained in the genesis of vesico-uterine fistula. The tone of the uterine isthmus being superior to that of the bladder muscle, the flow is one-way, namely from the uterus to the bladder. It is therefore a fistula opening into the uterine isthmus. Those that emerge above the uterine isthmus function in both directions.

The modes of discovery are variable and are dependent on the size and location of the fistula. The symptomatology may also vary over time in the same patient. The Youssef's syndrome in its original description, classically associates cyclical hematuria or menouria, amenorhea and urinary continence in the immediate aftermath of a lower segmental caesarean section. But this typical picture is very rare, urinary continence is rarely observed, imperious micturition are sometimes noted [14, 16, 17]. Urinary incontinence occurs if the level of the vesicouteine fistula is at or below the internal os or if the os is incompetent. Classically, $42 \%$ of the patients in this study had Youssef's syndrome. The intermittent leaking urine per vagina that disappear during menstruation (due to uterine congestion that compresses the fistula) found in $38 \%$ of our patients. Painless, total, cyclic and monthly hematuria during the period of menstruation found in $30.8 \%$ cases of the present study. A secondary amenorrhea without any hormonal disorder, in fact it is a pseudo-amenorrhea, the menses flow being eliminated by the bladder during urination (the woman urinates her menses) and also the fortuitous discovery of a vesicouterine fistula during a hysterography carried out, for example, during a sterility check. However patients of this study were all symptomatic and had signs that varied from one to another (Table 2).

During the speculum examination, the bladder filling with physiological saline supplemented with methylene blue allowed to see blue from the cervix in 21 cases, signifying the communication between both uterine and bladder cavities. This test however does not show directly the fistulous tract nor its specific location. The absence of leakage does not invalidate the diagnosis. Although clinical signs can lead to the diagnosis of vesicouterine fistula in most cases, it remains very important to confirm the diagnosis by paraclinical exams.

Retrograd cystography allows theoretically to see the fistulous tract. But it can be normal and it rarely show the uterine cavity. At hysterography, the communication between bladder and uterus appears on the profile snapshot. Cystoscopy may not visualize the communication orifice generally buried in an oedematous and echymotic zone. Hysteroscopy studies the cervical canal, the scar of the caesarean section and the endometrium and allows its biopsy. Intravenous urography can show the fistula but distinguishing vesicovaginal and vesicouterine fistula may be difficult. It appreciates the course of the ureters and the possible repercussion on the upper urinary tract. Radiographically, both hysterography and cystography are commonly used to diagnose this pathology [11, 18, 19]. In our context, these two tests were sufficient to establish the diagnosis of vesico-uterine fistula in all cases. In a very well equipped specialized department, when there are problems with visualization of the fistula, investigations like computed tomography (CT) and magnetic resonance imaging (MRI) should be used for diagnosis. These are used because give more information about the precise topography of the 
fistulous tract and surrounding anatomy [11, 16] and especially when a tumor etiology is suspected.

The treatment of obstetric fistula must be before any restorative and functional without compromising the vital prognosis, should be adapted not only to the age of the patients mostly young, but also to their cultural environment with only concern, a social and family reintegration in very good conditions. Vesicouterine fistula may heal spontaneously with involution of the puerperal uterus by bladder cathetrization for at least 4-8 weeks is indicated when the fistula is discover just after delivery and is small. Spontaneous healing may occur in $5 \%$ of cases $[9,20]$. When it does not, continuous hormonal therapy can be given to suppress menstruation for 3-6 months as first line of therapy [9]. But surgery is the mainstay and definitive treatment of vesicouterine fistulas. Surgical repair of vesicouterine fistulas are performed by different approaches (depending upon the surgical experience) which include the vaginal, transvesical-retroperitoneal and transperitoneal access which is considered the most effective with the lowest relapse rate [9]. The diagnostic delay (average time of 6 years in this study), the ease of execution and the better exposure of the lesions made us prefer transperitoneal laparotomy with opening the vesicouterine space in the first line. Bladder and uterus have been dissected and separated, fistulous orifices resected then uterine and vesical sutures were performed with resorbable thread. The interposition of the peritoneum which was not systematic was necessary in 2 cases. The existence of associated lesions, difficulties of exposure, menopause or proximity to menopause justifies the hysterectomy with closure of the fistulous vesical orifice that we carried out in 4 cases. Other relatively recent techniques have been proposed, namely cystoscopic fulguration consisting of endoscopic electrocoagulation (the endoscopic treatment may be effective in treating small vesicouterine fistulas) the vesical orifice of the fistula [10], as well as laparoscopic treatment of vesicouterine fistula at the vesicocervical level $[17,21]$. A urinary catheter is essential and a radiographic control desirable three to four weeks after the cure.

We found satisfactory results. Functionally, we observed dryness, urinary continence and the reappearance of vaginal menstruation in all cases including 3 after a second operation. To date, among the patients operated, there were 8 who had been able to lead a pregnancy. This is a prognosis that could improve in a few years reflecting a good beginning of family reinsertion. In the literature the pregnancy rate after repair is $31.25 \%$ to $37.55 \%$ with a rate of live birth of $25 \%[9,20]$.

\section{Conclusion}

Vesicouterine fistula is a rare complication obstetrical and very often iatrogenic, related to childbirth. The good prognosis after repair should not be a success and make forget that this pathology seriously affect the quality of the patient's life for several years before diagnosis. It is rather the success of permanent and quality prevention that must remain privileged. And the different prevention measures are well coded and well known.

\section{References}

[1] Browning A, Whiteside S: Characteristics, management, and outcomes of repair of rectovaginal fistula among 1100 consecutive cases of female genital tract fistula in Ethiopia. Inter J Gyn Obstet 2015; 131: 70-3.

[2] Dumurgier C, Falandy L: La chirurgie des fistules obstétricales: Bull Acad Natle Med 2012; 196,8: 1535-56.

[3] Ndiaye P, Kinin AP, Adama F, Idrissa A, Tal-Dia A. Fistule urogénitale d'origine obstétricale: Coùt de la prise en charge à l'hopital national de Niamey (Niger). Revue d'Epidémiologie et de Santé Publique 2009; 57, 5: 374-9.

[4] Konan KP, Fofana A, Kramo NF, Vodi CC, Gowe EE, Dekou $\mathrm{AH}$ et al. Les fistules urogénitales dans le service d'urologie du CHU de Cocody. Aspects évolutifs de 1990 à 2010. Uro'Andro 2015; 1,3: 157-61.

[5] Kochakarn W, Pummangura W. A new dimension in vesicovaginal fistula management: An 8 years experience at Ramathibodi hospital. Asian J Surg 2007; 30: 267-71.

[6] Creanga AA, Genadry RR. Obstetrics fistulas: a clinical review. Int J gynaecol Obstet 2007; 99 suppl1:S40-6.

[7] Raashid Y, Tmajeed T, Mmajeed N, Shahzad N. Iatrogenic vesicovaginal fistula. J Coll Physicians Surg Pak 2010;20 (2): 436-8.

[8] Majeed SM, subhani SS. An unusual case of Youssef's syndrome (vesicouterine fistula) and its relationship with placenta percreta. J coll physicians surg pak 2007; 17 (3): 1689.

[9] Dilruba A, Salahuddin S, Ferdousi I, Saria T. Successful pregnancy following repair of vesicouterine fistula following repeat caesarean section: A case Report. BSMMU J 2012; 5(1): 76-8.

[10] Drissi M, Karmouni T, Tazi K, El Khader K, Koutani A, Ibn Attya A et al. Les fistules vésico-uterines: une expérience de 17 ans. Prog Urol 2008;18,3: 173-6.

[11] Shanmugasundaram R, Gopalakrishnan G, Kebre SN. Youssef's syndrome: is there a better way to diagnose? Indian J Urol 2008; 24(2): 269-70.

[12] Onsrud M, Sjoveian S, Mukwege D. Cesarean delivery-related fistulae in the Democratic Republic of Congo. Int J Gynecol Obstet 2011; 114: 10-4.

[13] Rajamaheswari N, Chhikara AB. Vesicouterine fistulae: our experience of 17 cases and literature review. Int Urogynecol J 2013; 24: 275-9.

[14] Ikechebelu IJ, Ugboaji JO, Okeke CF. Postcesarean vesicoterine (Youssef syndrome): reporte of two cases. J Obstet Gynaecol Research 2011; 37, 7: 912-5.

[15] Shephard NS, Lengmang SJ. The missig foley catheter: an unusual finding in vesicouterine fistula. J Surg Case Rep 2013(12): rjt115. 
[16] Wiborg MH, Walter S. Fistula as cause of cyclic haematuria in women. Ugeskr Laeger 2014 Dec 15; 176 (25A). Pii: v09120556.

[17] Tasdemir N, Abali R, Celik C, Yazici CM, Akkus D. The use of and anterior abdominal wal peritoneal flap in the laparoscopic repair of vesicouterine fistula. Int Surg 2014; 99(5): 673-6.

[18] Fenkei IV, Demirbas M, Oztekin O. Sonohysterography in evaluation of Youssef's sydrome. Int Urogynecol J 2010; 21(5): 607-8.
[19] Gharoro EP, Enabudoso EJ, Gharoro EE. Sonographic appearance of youssef's syndrome: a case reported and literature review. Open J Obstet Gynecol 2013; 3:553-5. doi:10.4236/ojog.2013.37099.

[20] Hadzi-Djokie JB, Pejcic TP, Colovic VC. Vesicouterine fistulas: report of 14 cases. BUJ Int 2007; 100(6): 1361-3.

[21] Aminsharifi A, Afsar F, Shirazi MK. Int J Gynecol Obstet 2014; 124(2): 148-50. 